\title{
Contribution to the lichen biota of the Pogórze Wiśnickie foothills (Carpathians)
}

\author{
LUCYNA ŚLIWA \\ Laboratory of Lichenology, W. Szafer Institute of Botany, Polish Academy of Sciences \\ Lubicz 46, PL-31-512 Kraków, 1.sliwa@botany.pl
}

Śliwa L.: Contribution to the lichen biota of the Pogórze Wiśnickie foothills (Carpathians). Acta Mycol. 45(2): 219-230, 2010.

The Pogórze Wiśnickie foothills are situated in close vicinity to the Kraków agglomeration and is highly influenced by human activity. Lichen studies in the area revealed 163 species so far. A current checklist of the lichen biota of the territory is provided with numerous new regional records, e.g., Bacidina sulphurella, Evernia prunastri, Fuscidea pusilla, Lecanora albellula, Lepraria ecorticata, Mycobilimbia epixanthoides, Ramalina farinacea, R. fastigiata, Ropalospora viridis, Verrucaria praetermissa and V.tectorum.

Key words: lichens, biodiversity, lichenized fungi, new records, Poland

\section{INTRODUCTION}

The Carpathians are among the best known areas in Poland concerning the lichen biota (Bielczyk 2006), with respective literature comprising ca 300 references (Bielczyk 2003). The Carpathian foothills, however, though being close to the centres of lichenological investigations of southern Poland, remain poorly explored both historically and geographically. Among the foothills the most thoroughly explored areas are: Pogórze Rożnowskie and Ciężkowicke (Kozik 1970, 1976, 1977; Czwórnóg, Śliwa 1995), Pogórze Spisko-Gubałowskie (Kiszka 1985), Pogórze Przemyskie (Kiszka, Piórecki 1991; Kiszka 2002a, b). Fragmentary data originate from Pogórze Bukowskie (Rydzak 1955), Pogórze Wielickie (Kiszka 1996a), Pogórze Dynowskie (Krzewicka, Śliwa 2000), and Pogórze Śląskie (Leśniański 2001) foothills. The latter regions as well as others (e.g., Pogórze Strzyżowskie, Rzeszowskie and Jasielskie foothills) are in urgent need of complex investigations especially with rapid changes of environmental conditions caused by human activity and the influence of changing climate. Due to these same reasons some of the early investigations require reexamination. 
In the years 1998-2000 a lichen survey was carried out aimed at a more comprehensive exploration of the Pogórze Wiśnickie foothills with special emphasis on protected areas. The project included lichenological training for students of the Jagiellonian University and resulted in some joint publications (Śliwa et al. 2001; Śliwa, Krzewicka 2004). Part of the results were also published by Stolarczyk (2003). This paper presents a part of a summary of the research as a contribution to the knowledge of the lichen biota of this interesting area highly influenced by human activity.

\section{STUDY AREA}

The Carpathian Foothills form a transitional area that is located between the Beskid in the south and the sub-Carpathian basins in the north, and the landscape is affected by three main subsystems: abiotic, biotic and anthropogenic (Drużkowski 1998). The Pogórze Wiśnickie foothills constitute the easternmost edge of the Pogórze Zachodniobeskidzkie foothills of the Western Carpathians (Kondracki 1989, 2001). Neighbouring areas are the Pogórze Wielickie foothills to the west and the Pogórze Rożnowskie foothills to the east. The territory includes several protected areas (e.g., the Bukowiec Nature Reserve, Kamień Grzyb and Kamienie Brodzińskiego protected sandstone tors) as well as areas of special concern (Wiśnicko-Lipnicki Landscape Park). In general it is rich in natural values represented by forested hills and unique landscape formations such as sandstone tors. Forest communities occupying the area were characterised by Stachurska (1998a, b).

On the other hand the Pogórze Wiśnickie foothills is under the visible impact of human activity; some parts of the area are strongly urbanized and influenced by industrial and transportation emissions of Krakow and surrounding towns. Details of the environmental transformation of the whole area of the Carpathians Foothills due to natural and anthropogenic factors is presented by Drużkowski (1998), who evaluated the transformation as moderate at present.

\section{MATERIAL AND METHODS}

Lichens were collected in the years 1998-2000 at 32 sites located in the Fe ATPOL gird square system (acc. to Cieśliński, Fałtynowicz 1993). Each $100 \times 100 \mathrm{~km}(\mathrm{Fe})$ plot was divided into $10 \times 10 \mathrm{~km}$ units numbered from 00 to 99 , and then into $2 \times 2$ $\mathrm{km}$ subunits also numbered from 00 to 99 - the numbers follow one the other in the list of collecting sites. All habitats and substrates were explored. Lichens were identified using routine microscopic and laboratory techniques. When necessary the TLC analyses was performed in solvent system A or/and C (methods followed Orange et al. 2001).

Voucher specimens are available at KRA and/or KRAM herbaria. Nomenclature basically follows Santesson et al. (2004) and Diederich et al. (2010). 
Lichen collecting sites in the Pogórze Wiśnickie foothills: 1 - Cichawka village, S slope of a hill along the road, Fe 8233; forest with Pinus sylvestris and Quercus robur, and road side trees, 8 May 1998, L. Śliwa collection numbers 539-561; 2 Cichawka village, near the church, Fe 8233; stream valley with Alnus glutinosa, rocks and stones, 8 May 1998, L. Śliwa 562-592; 3 - Wieruszycka village, along Stradomka stream, Fe 8232, shrubs with Alnus glutinosa, 19 May 1998, L. Śliwa 593-614; 4 Cichawka stream headwaters, Fe 8234, mixed forest with Fagus sylvatica, Carpinus betulus, Pinus sylvestris and Abies alba, 19 May 1998, L. Śliwa 615-662; 5 - KrólówkaSkotnica village, along stream, Fe 8331, mixed forest and concrete constructions; 2 June 1998, L. Śliwa 663-728; 6 - Królówka-Uzbornia village, Fe 8321, trees along road side and stream, 2 June 1998, L. Śliwa 663-728; 7 - E part of Łapanów village, road to Muchówka, Fe 8231, road side trees of Fraxinus excelsior, 3 June 1998, L. Śliwa 662-796; 8 - Łapanów-Rogatka village, Fe 8232, trees along stream and road side trees of Populus spp., 3 June 1998, L. Śliwa 797-837; 9 - Wola Wieruszycka village, Fe 8222, Salix spp. trees along stream and fruit trees, 17 June 1998, L. Śliwa 838-873; 10 - Chrostowa Góra Mt., Fe 8212, mixed forest with Quercus robur, Fagus silvatica and Pinus sylvestris, 17 June 1998, L. Śliwa 874-880; 11 - Chrostowa village, Fe 8212, road side trees, 17 June 1998, L. Śliwa 881-898; 12 - Dąbrowica village, park by the school, Fe 8211, 17 June 1998, L. Śliwa 899-910; 13 - Forest between Dołuszyce and Pogwizdów and Kobylany villages, S slope, Fe 7341, mixed forest with Fagus sylvatica, Quercus robur, Carpinus betulus, Betula pendula, and Pinus sylvestris, 21 April 1999, L. Śliwa 975-992; 14 - Road from Dołuszyce to Pogwizdów village, along a stream, Fe 7340, young forest with Alnus glutinosa, Fraxinus excelsior and Quercus robur, 21 April 1999, L. Śliwa 993-1016; 15 - Bukowiec Nature Reserve, alt. 430-460 m, Fe 8442, mixed forest and shaded streams, 21 May 1999 and 22 Sept. 1999, L. Śliwa 1017-1075; 16 - Tymowa Górna village, Fe 9342, road side trees, 22 Sept. 1999, L. Śliwa 1076-1082; 17 - Lipnica Murowana village, road to Nowy Wiśnicz town, Fe 8334, Salix spp., 1 March 2000, L. Śliwa 1083; 18 - Stary Wiśnicz town, road junction, Fe 8303, concrete post, 24 March 2000, L. Śliwa 1084; 19 - Lipnica Górna village, road to Rajbrot, Fe 8344, Betula pendula, 24 March 2000, L. Śliwa 1085; 20 - E part of Kobyle village, road side trees, Fe 8400, Populus spp., 24 March 2000, L. Śliwa 1086-1087; 21 - Krasa Góra village, road side, Fe 8420, 24 March 2000, L. Śliwa 1088; 22 - Muchówka village, road to Żegocina, Fe 8342, 24 March 2000, L. Śliwa 1089-1093; 23 - NW slope of the hill “409 m a.s.l” near Mt. Paprotna Góra, Fe 8342, mixed forest with rocks, 7 May 2000, L. Śliwa 1094-1104; 24 - Hill by road to Rajbrot village, near Mt. Paprotna Górna, Fe 8342; mixed forest with Pinus sylvestris and Betula pendula, 7 Maj 2000, L. Śliwa 1105-1114; 25 - "Kamień Grzyb" stone in Bigorzówka village near Raciechowice, Fe 9102, sandstone, 7 May 2000, L. Śliwa 1115-1126; 26 - "Diabelski Kamień" stone in Smykan village near Szczyrzyce, Fe 9122, sandstone, 7 May 2000, L. Śliwa 1127-1159; 27 - Forest S of Sobolów village, Fe 8215, mixed forest with Quercus robur, Fagus sylvatica and Picea abies, 5 June 2000, L. Śliwa 1160-1182; 28 - Mt. Góra Łysa between Zonia and Nieprzaśna villages, near quarry, Fe 8214, forest with Fagus sylvatica and Pinus sylvestris, 5 June 2000, L. Śliwa 1183-1190; 29 - Wichraź village near Zagrody, stream valley, Fe 8224, forest ridge, 20 June 2000, L. Śliwa 1191-1212; 30 - Sieradzka village, stream valley, Fe 8223, forest with Fraxinus excelsior, Alnus glutinosa and Carpinus betulus, 20 June 2000, L. Śliwa 1213-1234; 31 - Dziekanowice village, along Raba River, Fe 8014, 
sandstone outcrops, 29 June 2000, L. Śliwa 1235-1257; 32 - Leszczyna village, W of junction with the road to Rozstajnie village, Fe 8234, road side trees, mainly Salix spp., 30 June 2000, L. Śliwa 1258-1267.

\section{RESULTS AND DISCUSSION}

The checklist of taxa presented below (Tab. 1) summarizes all known data concerning the lichen biota of the Pogórze Wiśnickie foothills.

Table 1

Lichenized fungi recorded in the Pogórze Wiśnickie foothills until the present

Source of information: 1 - Śliwa et al. (2001); 2 - Stolarczyk (2003); 3 - Śliwa \& Krzewicka (2004); 4 herbarium material, leg. Sliwa (coll. no.). Bark of trees: Ab - Abies alba, Ap - Acer platanoides, Ae - Aesculus hippocastanum, $\mathrm{Al}$ - Alnus glutinosa, $\mathrm{B}$ - Betula pendula, $\mathrm{Cb}$-Carpinus betulus, $\mathrm{Fs}$ - Fagus sylvatica, $\mathrm{Fr}$ - Fraxinus excelsior, $\mathrm{Jr}$ - Juglans regia, Ma - Malus domestica, Lar - Larix decidua, $\mathrm{Ps}-$ Pinus sylvestris, Po - Populus spp., Pr - Prunus spp., Qr - Quercus robur, Sa - Salix spp., Tc - Tilia cordata .

\begin{tabular}{|c|c|c|}
\hline Name of species & Substrate & Source of information \\
\hline Acarospora fuscata (Schrad.) Th.Fr. & $\begin{array}{l}\text { sandstone rocks, } \\
\text { concrete }\end{array}$ & $1 ; 2$ \\
\hline Acrocordia gemmata (Ach.) A. Massal. & Po & $2 ; 4(609,1260)$ \\
\hline $\begin{array}{l}\text { Amandinea punctata (Hoffm.) Coppins \& } \\
\text { Scheid. }\end{array}$ & $\begin{array}{l}\text { Po, Tc, Fr, Sa, Qr, B, } \\
\text { Ma, Jr }\end{array}$ & $\begin{array}{l}2 ; 4(540 b, 547,605,597, \\
720,735,751,772,826 a, \\
832 a, 838,847,883,884, \\
900)\end{array}$ \\
\hline $\begin{array}{l}\text { Anisomeridium polypori (Ellis \& Everh.) } \\
\text { M.E. Barr }\end{array}$ & Po & $2 ; 4(857,996,1216 b, 1086)$ \\
\hline Arthonia radiata (Pers.) Ach. & $\mathrm{Fs}, \mathrm{Al}, \mathrm{Cb}$ & $\begin{array}{l}4(564,659,703,705,998 \\
1005 b, 1216 a, 1221 b, 1223 \\
1228)\end{array}$ \\
\hline Arthonia spadicea Leight. & $\mathrm{Cb}$ & $4(563)$ \\
\hline Aspicilia calcarea (L.) Mudd & $\begin{array}{l}\text { calcareous stones, } \\
\text { concrete, tile }\end{array}$ & 2 \\
\hline Bacidia trachona (Ach.) Lettau & sandstone rocks & 1 \\
\hline Bacidina sulphurella (Samp.) comb. ined. & $\mathrm{Fs}, \mathrm{Cb}$ & $4(598,612,1060,1061)$ \\
\hline Baeomyces rufus (Huds.) Rebent. & soil, sandstone rocks & $1 ; 2 ; 3 ; 4(621,977,1112)$ \\
\hline Bilimbia sabuletorum (Schreb.) Arnold & $\begin{array}{l}\text { sandstone, concrete, } \\
\text { bryophytes }\end{array}$ & 2 \\
\hline Buellia griseovirens (Sm.) Almb. & Po, Ma & $2 ; 4(726,754,849 b, 1003)$ \\
\hline Caloplaca citrina (Hoffm.) Th.Fr. & $\begin{array}{l}\text { concrete, sandstone } \\
\text { rocks, asbestos tile }\end{array}$ & $2 ; 4(671,795,1093)$ \\
\hline Caloplaca vitellinula auct. & concrete & 2 (needs rev.) \\
\hline $\begin{array}{l}\text { Caloplaca decipiens (Arnold) Blomb. \& } \\
\text { Forssell }\end{array}$ & $\begin{array}{l}\text { concrete, asbestos } \\
\text { tile, tile }\end{array}$ & $2 ; 4(683,714,1192)$ \\
\hline Caloplaca holocarpa (Ach.) A.E. Wade & $\begin{array}{l}\text { concrete, sandstone } \\
\text { rocks, tile }\end{array}$ & $2 ; 4(712)$ \\
\hline Caloplaca velana (A. Massal.) Du Rietz & concrete & $4(674,863 b, 866)$ \\
\hline Caloplaca saxicola (Hoffm.) Nordin & concrete & 2 \\
\hline Candelaria concolor (Dicks.) Stein & Po & $2 ; 4(665 a)$ \\
\hline Candelariella aurella (Hoffm.) Zahlbr. & $\begin{array}{l}\text { concrete, sandstone } \\
\text { rocks, tile }\end{array}$ & 2 \\
\hline Candelariella coralliza (Nyl.) H. Magn. & sandstone rocks & 2 \\
\hline Candelariella reflexa (Nyl.) Lettau & $\begin{array}{l}\text { Ma, Po, Sa, Fr, Tc, } \\
\text { Ae }\end{array}$ & $\begin{array}{l}2 ; 4(599,665 a, 740,818 \\
1007,1077)\end{array}$ \\
\hline Candelariella vitellina (Hoffm.) Müll. Arg. & sandstone rocks, tile & $2 ; 4(713,724)$ \\
\hline Candelariella xanthostigma (Ach.) Lettau & Po, Sa, Tc, Fr, Ma, Jr & $2 ; 4(1078)$ \\
\hline
\end{tabular}


Table 1 - cont.

\begin{tabular}{|c|c|c|}
\hline Chaenotheca chrysocephala (Ach.) Th.Fr. & Sa & $4(684,848)$ \\
\hline $\begin{array}{l}\text { Chaenotheca ferruginea (Turner \& Borrer) } \\
\text { Mig. }\end{array}$ & $\mathrm{Al}, \mathrm{Fs}, \mathrm{Ps}$ & $2 ; 3 ; 4(677,1008,1010)$ \\
\hline Chaenotheca furfuracea (L.) Tibell & soil, roots of trees & $4(575,1198)$ \\
\hline Chaenotheca xyloxena Nádv. & wood & 3 \\
\hline Cladonia caespiticia (Pers.) Flörke & soil & $\begin{array}{l}1 ; 2 ; 4(551,556,1161, \\
1172)\end{array}$ \\
\hline Cladonia chlorophaea (Sommerf.) Spreng. & $\begin{array}{l}\text { soil, sandstones, Ps, } \\
\text { B, Sa, Fr, Ma }\end{array}$ & $1 ; 2 ; 4(988)$ \\
\hline Cladonia coniocraea (Flörke) Spreng. & $\begin{array}{l}\text { soil, wood, bark of } \\
\text { trees }\end{array}$ & $\begin{array}{l}1 ; 2 ; 3 ; 4(585,641,855, \\
992)\end{array}$ \\
\hline Cladonia digitata (L.) Hoffm. & Ps & $1 ; 2$ \\
\hline Cladonia fimbriata (L.) Fr. & soil & $\begin{array}{l}2 ; 4(733,831,985,1181, \\
1231)\end{array}$ \\
\hline Cladonia macilenta Hoffm. & B, Ps; wood, soil & $1 ; 2 ; 3 ; 4(890)$ \\
\hline Cladonia ochrochlora Flörke & Ps; wood & $1 ; 2 ; 3$ \\
\hline Cladonia pleurota (Flörke) Schaer. & sandstone rocks & 1 \\
\hline Cladonia parasitica (Hoffm.) Hoffm. & soil & $2 ; 3$ (needs rev.) \\
\hline Cladonia pyxidata (L.) Hoffm. & soil & 2 \\
\hline Cladonia rei Schaer. & soil & 2 (needs rev.) \\
\hline Cladonia squamosa Hoffm. & Ps & 2 \\
\hline Cladonia subulata (L.) F.H. Wigg. & soil & 2 \\
\hline $\begin{array}{l}\text { Coenogonium pineti (Ach.) Lücking \& } \\
\text { Lumbsch }\end{array}$ & $\mathrm{B}, \mathrm{Qr}$ & $\begin{array}{l}2 ; 3(\text { sub Dimerella diluta }) ; 4 \\
(557 b, 994 b, 1109,1191)\end{array}$ \\
\hline Collema tenax (Sw.) Ach. & soil & $4(1089)$ \\
\hline Dibaeis baeomyces (L.f.) Rambold \& Hertel & soil & $2 ; 4(560,572)$ \\
\hline Diploschistes scruposus (Schreb.) Norman & sandstone rocks & 1 \\
\hline Evernia prunastri (L.) Ach. & Po & $4(545,781,1267)$ \\
\hline Fuscidea pusilla Tønsberg & $\mathrm{Al}$ & $4(704,1220 a, 1221 d)$ \\
\hline Graphis scripta (L.) Ach. & $\mathrm{Fs}, \mathrm{Cb}$ & $\begin{array}{l}1 ; 3 ; 4(567,649,650,661 b, \\
696,697,702,1005 a, 1225 a, \\
1230 b)\end{array}$ \\
\hline $\begin{array}{l}\text { Hypocenomyce caradocensis (Nyl.) P. James } \\
\& \text { Gotth. Schneid. }\end{array}$ & Ps & $2 ; 3$ \\
\hline Hypocenomyce scalaris (Ach.) M. Choisy & $\begin{array}{l}\text { B, Fs, Fr, Ma, Ps; } \\
\text { wood }\end{array}$ & $\begin{array}{l}2 ; 3 ; 4(552,648,727,776, \\
825,854,876,909 b, 975, \\
1013,1098,1168)\end{array}$ \\
\hline Hypogymnia physodes (L.) Nyl. & $\begin{array}{l}\text { Ps, Po, Fr, Qr, Ma, } \\
\text { Sa, Pr; tile }\end{array}$ & $\begin{array}{l}2 ; 3 ; 4(576,602,634,687 \\
743,817,851 c, 1227)\end{array}$ \\
\hline Hypogymnia tubulosa (Schaer.) Hav. & $\mathrm{Ma}$ & $2 ; 4(1217)$ \\
\hline Imshaugia aleurites (Ach.) S.L.F. Meyer & $\mathrm{B}$ & $4(1104)$ \\
\hline Lecania cyrtella (Ach.) Th.Fr. & $\mathrm{Al}, \mathrm{Sa}, \mathrm{B}$ & $4(755,816,821 a)$ \\
\hline Lecanora albellula Nyl. & Po, Sa, Fs, Qr, Ma & $\begin{array}{l}4(601,610,678,746,757, \\
1173 a)\end{array}$ \\
\hline Lecanora albescens (Hoffm.) Flörke & concrete & $2 ; 4(1202)$ \\
\hline Lecanora carpinea (L.) Vain. & Fr, Sa & $4(681,815)$ \\
\hline Lecanora chlarotera Nyl. & Po & $4(736)$ \\
\hline Lecanora conizaeoides Crombie & $\begin{array}{l}\text { Ps, B, Sa, Qr, Po, Fs, } \\
\text { Al, Ap, Lar, Cb, Ma, } \\
\text { Pr; wood }\end{array}$ & $\begin{array}{l}1 ; 2 ; 3 ; 4(553,565,614,625, \\
652,676,688 a, 773,819, \\
862,888,904,909 a, 986)\end{array}$ \\
\hline Lecanora dispersa (Pers.) Sommerf. & $\begin{array}{l}\text { concrete, sandstone } \\
\text { rocks, bark of trees, } \\
\text { asbestos tile, tile }\end{array}$ & $\begin{array}{l}2 \text { (incl. L. semipallida); } 4 \\
(760,796,867,1091,1201)\end{array}$ \\
\hline Lecanora expallens Ach. & $\begin{array}{l}\text { Po, Sa, Ap, Fr, Tc, } \\
\text { Qr, Ma; asbestos tile }\end{array}$ & 2 (needs rev.) \\
\hline Lecanora hagenii (Ach.) Ach. & wood, concrete & $4(794,1164 a, 1200)$ \\
\hline Lecanora muralis (Schreb.) Rabenh. & sandstone rocks & $2 ; 4(692)$ \\
\hline Lecanora polytropa (Hoffm.) Rabenh. & sandstone rocks & 1 \\
\hline Lecanora pulicaris (Pers.) Ach. & Sa, Al, Qr, Ma; wood & $2 ; 3 ; 4(655,680)$ \\
\hline Lecanora saligna (Schrad.) Zahlbr. & $\begin{array}{l}\text { Po, Sa, Fs, Qr, Ma; } \\
\text { wood }\end{array}$ & $\begin{array}{l}2 ; 4(624,672,834,1014, \\
1195,1257,1264)\end{array}$ \\
\hline
\end{tabular}


Table 1 - cont.

\begin{tabular}{|c|c|c|}
\hline Lecanora semipallida $\mathrm{H}$. Magn. & concrete & $\begin{array}{l}4(701,711,729 a, 1084, \\
1090,1164)\end{array}$ \\
\hline Lecanora symmicta (Ach.) Ach. & wood & $4(851 a)$ \\
\hline Lecanora varia (Hoffm.) Ach. & wood & $2 ; 4(695)$ \\
\hline Lecidea fuscoatra (L.) Ach. & sandstone rocks & 1 \\
\hline Lecidella elaeochroma (Ach.) M. Choisy & $\mathrm{Sa}, \mathrm{B}$ & 2 \\
\hline Lecidella stigmatea (Ach.) Hertel \& Leuckert & concrete, tile & $\begin{array}{l}2 ; 4(653,710,797,832 b, \\
833 a, 845,1092)\end{array}$ \\
\hline Lepraria borealis Lothander \& Tønsberg & $\begin{array}{l}\text { sandtone rocks, } \\
\text { bryophytes }\end{array}$ & $4(1119 a)$ \\
\hline Lepraria caesioalba (de Lesd.) J.R. Laundon & $\begin{array}{l}\text { sandtone rocks, soil, } \\
\text { bryophytes }\end{array}$ & $1 ; 4(1119 b)$ \\
\hline Lepraria eburnea J.R. Laundon & $\mathrm{Sa}$ & $4(774 a, 1222,1262)$ \\
\hline Lepraria ecorticata (J.R. Laundon) Kukwa & sandstone rock & $4(1132)$ \\
\hline Lepraria elobata Tønsberg & $\begin{array}{l}\mathrm{Al}, \mathrm{Sa}, \mathrm{Ma}, \mathrm{B}, \mathrm{Fs} \\
\text { soil, rocks }\end{array}$ & $\begin{array}{l}3 ; 4(557 a, 586,643,669 \\
774 b, 813,982,1096,1110 \\
1113,1162,1188,1204 \\
1252)\end{array}$ \\
\hline Lepraria incana (L.) Ach. & $\begin{array}{l}\text { Po, Fr, Sa, Fs, B, Tc; } \\
\text { sandstone rocks }\end{array}$ & $\begin{array}{l}\text { 1; 3; } 4(676,689,899,987, \\
1082,1133,1144,1207 \\
1263)\end{array}$ \\
\hline Lepraria jackii Tønsberg & bryophytes & $4(1169,1171)$ \\
\hline Lepraria lobificans $\mathrm{Nyl}$. & $\begin{array}{l}\mathrm{Sa}, \mathrm{Tc}, \mathrm{Al}, \mathrm{Fs}, \mathrm{Ps} ; \\
\text { wood, sandstone } \\
\text { rocks }\end{array}$ & $\begin{array}{l}1 ; 3 ; 4(581,608,734,774 c, \\
839,978,995,1094,1105, \\
1114,1205,1225 b, 1230 a \\
1256)\end{array}$ \\
\hline Lepraria membranacea (Dicks.) Vain. & sandstone rocks & $1 ; 2 ; 4(1136,1147 b, 1238)$ \\
\hline Lepraria neglecta (Nyl.) Lettau & sandstone rocks & $1 ; 4(1119 c, 1147 a)$ \\
\hline Lepraria vouauxii (Hue) R.C. Harris & $\mathrm{Sa}, \mathrm{Po}, \mathrm{Ma}$ & $\begin{array}{l}4(546,632,764,836,887, \\
893)\end{array}$ \\
\hline Melanelixia fuliginosa (Duby) O. Blanco et al. & Po, Ap, Ae, Ma & $2 ; 4(647,678,715)$ \\
\hline $\begin{array}{l}\text { Melanelixia subargentifera (Nyl.) O. Blanco } \\
\text { et al. }\end{array}$ & $\mathrm{Sa}$ & $4(778,1258)$ \\
\hline $\begin{array}{l}\text { Melanelixia subaurifera (Nyl.) O. Blanco et } \\
\text { al. }\end{array}$ & Qr, Ae & $2 ; 4(567)$ \\
\hline $\begin{array}{l}\text { Melanohalea elegantula (Zahlbr.) O. Blanco } \\
\text { et al. }\end{array}$ & $\mathrm{Sa}$ & 2 (sub Melanelia incolorata) \\
\hline $\begin{array}{l}\text { Melanohalea exasperatula (Nyl.) O. Blanco } \\
\text { et al. }\end{array}$ & Tc, Sa, Fr, Ma; tile & $2 ; 4(593,890)$ \\
\hline Micarea botryoides (Nyl.) Coppins & bryophytes, wood & $\begin{array}{l}3 \text { (cf. Czarnota 2007); } 4 \\
(592,699)\end{array}$ \\
\hline Micarea denigrata (Fr.) Hedl. & sandstone, wood & $2 ; 3 ; 4(729 b, 1097)$ \\
\hline Micarea lignaria (Ach.) Hedl. & B; soil & $4(577,1102)$ \\
\hline Micarea micrococca (Körb) Coppins & $\mathrm{Cb}, \mathrm{Ps}$ & $4(639 c, 1232)$ \\
\hline Micarea misella (Nyl.) Hedl. & rotting wood & $3 ; 4(1182,1197 b)$ \\
\hline $\begin{array}{l}\text { Micarea peliocarpa (Anzi) Coppins \& R. } \\
\text { Sant. }\end{array}$ & sandstone rock & 1 \\
\hline Micarea prasina Fr. & rotting wood & $\begin{array}{l}3 ; 4(615 b, 627 b, 679, \\
1108 a)\end{array}$ \\
\hline Micarea viridileprosa Coppins \& v.d. Boom & wood & $\begin{array}{l}3 \text { (cf. Czarnota 2007); } 4 \\
(1108 b)\end{array}$ \\
\hline $\begin{array}{l}\text { Mycobilimbia epixanthoides (Nyl.) Hafellner } \\
\text { \& Türk }\end{array}$ & $\mathrm{Sa}$ & $\begin{array}{l}4(623,666,840,850,1081 \\
1083,1173 b)\end{array}$ \\
\hline Mycoblastus fucatus (Stirtan) Zahlbr. & $\mathrm{B}, \mathrm{Qr}$ & $2 ; 4(656)$ \\
\hline Parmelia omphalodes (L.) Ach. & sandstone rocks & 1 \\
\hline Parmelia saxatilis (L.) Ach. & sandstone rocks, Ma & $\begin{array}{l}1 ; 2 ; 3 ; 4(584,1009,1012, \\
1103)\end{array}$ \\
\hline Parmelia sulcata Taylor & $\begin{array}{l}\text { Po, Sa, Fr, Al, Tc, Qr, } \\
\text { Ma; tile }\end{array}$ & $\begin{array}{l}2 ; 4(571,594,628,717,752, \\
770,837 a, 849 a, 859,1004)\end{array}$ \\
\hline Parmelina tiliacea (Hoffm.) Hale & Tc, Po & $2 ; 4(768,802)$ \\
\hline Parmeliopsis ambigua (Wulfen) Nyl. & $\mathrm{Ma}, \mathrm{Al}$ & 2 \\
\hline $\begin{array}{l}\text { Pertusaria albescens (Huds.) M. Choisy \& } \\
\text { Werner }\end{array}$ & Ap, Sa & 2 \\
\hline
\end{tabular}


Table 1 - cont.

\begin{tabular}{|c|c|c|}
\hline Phaeophyscia nigricans (Flörke) Moberg & Po; concrete, tile & $2 ; 4(741)$ \\
\hline Phaeophyscia orbicularis (Neck.) Moberg & $\begin{array}{l}\mathrm{Po}, \mathrm{Sa}, \mathrm{Tc}, \mathrm{Ae}, \mathrm{Fr}, \\
\text { Cb, Jr, Ma; concrete, } \\
\text { asbestos tile }\end{array}$ & $\begin{array}{l}2 ; 4(603,747,767,780 a \\
873,892,898,905,1183)\end{array}$ \\
\hline Phaeophyscia sciastra (Ach.) Moberg & concrete & $4(693)$ \\
\hline Phlyctis argena (Spreng.) Flot. & $\mathrm{Tc}, \mathrm{Fr}, \mathrm{Ma}$ & $\begin{array}{l}1 ; 2 ; 4(542,635,790,812, \\
851 b, 901)\end{array}$ \\
\hline Physcia adscendens (Fr.) H. Olivier & $\begin{array}{l}\text { Po, Fr, Sa, Ma, } \\
\text { Cb, Jr, Ae, Tc, Al; } \\
\text { concrete }\end{array}$ & $\begin{array}{l}2 ; 4(539,640,706,730,748, \\
749,771,807,895,1015, \\
1079)\end{array}$ \\
\hline Physcia caesia (Hoffm.) Fürnr. & concrete, tile & $2 ; 4(758,786)$ \\
\hline Physcia dubia (Hoffm.) Lettau & concrete & $2 ; 4(737)$ \\
\hline Physcia stellaris (L.) Nyl. & Po & $2 ; 4(792)$ \\
\hline Physcia tenella (Scop.) DC. & Po, Tc, Fr, Sa, Ma, Jr & $\begin{array}{l}2 ; 4(595,596,620,691,719, \\
777,1226)\end{array}$ \\
\hline Physconia detersa (Nyl.) Poelt & Po & $4(779 a)$ \\
\hline Physconia distorta (With.) J.R. Laundon & Po & $4(763,783)$ \\
\hline Physconia enteroxantha (Nyl.) Poelt & $\mathrm{Po}, \mathrm{Sa}$ & $2 ; 4(742,779 b)$ \\
\hline Physconia grisea (Lam.) Poelt & $\mathrm{Po}, \mathrm{Sa}, \mathrm{Fr}$ & $2 ; 4(780 b)$ \\
\hline Physconia perisidiosa (Erichsen) Moberg & $\mathrm{Po}, \mathrm{Sa}$ & $2 ; 4(1011)$ \\
\hline Placynthiella dasaea (Stirt.) Tønsberg & $\begin{array}{l}\text { wood, Ps; sandstone } \\
\text { rocks }\end{array}$ & $2 ; 3 ; 4(1170,1178)$ \\
\hline $\begin{array}{l}\text { Placynthiella icmalea (Ach.) Coppins \& P. } \\
\text { James }\end{array}$ & Sa, Al, B; wood & $1 ; 2 ; 3 ; 4(1006,1166)$ \\
\hline $\begin{array}{l}\text { Placynthiella uliginosa (Schrad.) Coppins \& } \\
\text { P. James }\end{array}$ & Ma, Po, Fr; wood & $2 ; 3 ; 4(980)$ \\
\hline Placynthium nigrum (Huds.) Gray & sandstone rocks & $2 ; 4(664)$ \\
\hline Platismatia glauca W.L. Culb. \& C.F. Culb. & wood & $4(894)$ \\
\hline Porina aenea (Wallr.) Zahlbr. & Fs & $\begin{array}{l}2 ; 3 ; 4(639 a, 661 a, 993 \\
1221 c, 1224)\end{array}$ \\
\hline Porpidia tuberculosa (Sm.) Hertel \& Knoph & sandstone rocks & 2 \\
\hline Protoblastenia rupestris (Scop.) J. Steiner & $\begin{array}{l}\text { concrete, asbestos } \\
\text { tile }\end{array}$ & 2 \\
\hline $\begin{array}{l}\text { Protoparmelia hypotremella } \text { Herk, Spier \& } \\
\text { V.Wirth }\end{array}$ & $\mathrm{B}, \mathrm{Fr}$ & $2 ; 4(789)$ \\
\hline Pseudevernia furfuracea (L.) Zopf & $\begin{array}{l}\text { Ma; sandstone rocks, } \\
\text { tile }\end{array}$ & $\begin{array}{l}1 ; 2 ; 4(573 b, 756,897 \\
1218)\end{array}$ \\
\hline Punctelia subrudecta (Nyl.) Krog & $\mathrm{Sa}$ & $2 ; 4(630,787,902)$ \\
\hline Pyrenula nitida (Weigel) Ach. & Fs & $4(1177 a)$ \\
\hline Ramalina farinacea (L.) Ach. & $\mathrm{Fr}$ & $4(791)$ \\
\hline Ramalina fastigiata (Pers.) Ach. & Po & $4(803)$ \\
\hline Ramalina pollinaria (Westr.) Ach. & sandstone rocks & 2 \\
\hline Ropalospora viridis (Tønsberg) Tønsberg & $\mathrm{Cb}$ & $4(619,1221 e)$ \\
\hline Sarcogyne regularis Körb. & concrete & $2 ; 4(694,1206)$ \\
\hline Scoliciosporum chlorococcum (Stenh.) Vězda & $\begin{array}{l}\text { Po, Sa, Ma, Fs, Lar, } \\
\text { Fr, Ap, B, Ps, Qr, } \\
\text { Ab, Cb, Pr }\end{array}$ & $\begin{array}{l}1 ; 2 ; 3 ; 4(561,638,725,739, \\
759,766,853,877 a, 981)\end{array}$ \\
\hline Scoliciosporum umbrinum (Ach.) Arnold & sandstone & 2 \\
\hline Trapelia coarctata $(\mathrm{Sm}$.$) M. Choisy$ & $\begin{array}{l}\text { sandstone rocks, } \\
\text { pebbles }\end{array}$ & $\begin{array}{l}2 ; 3 ; 4(644,654,660,744, \\
991,1180,1184)\end{array}$ \\
\hline Trapelia involuta (Taylor) Hertel & sandstone rocks & $1 ; 3$ \\
\hline Trapelia obtegens (Th.Fr.) Hertel & sandstone rocks & $1 ; 2$ \\
\hline Trapelia placodioides Coppins \& P. James & sandstone rocks, tile & $1 ; 2$ \\
\hline $\begin{array}{l}\text { Trapeliopsis flexuosa (Fr.) Coppins \& P. } \\
\text { James }\end{array}$ & Qr, Ma, B; wood & $2 ; 3 ; 4(1163)$ \\
\hline $\begin{array}{l}\text { Trapeliopsis gelatinosa (Flörke) Coppins \& } \\
\text { P. James }\end{array}$ & soil & 1 \\
\hline Trapeliopsis granulosa (Hoffm.) Lumbsch & soil, wood & 3 \\
\hline $\begin{array}{l}\text { Trapeliopsis pseudogranulosa Coppins \& P. } \\
\text { James }\end{array}$ & $\begin{array}{l}\text { wood, soil, } \\
\text { bryophytes }\end{array}$ & $2 ; 3 ; 4(1001)$ \\
\hline $\begin{array}{l}\text { Trapeliopsis viridescens (Schrad.) Coppins \& } \\
\text { P. James }\end{array}$ & rotting wood & $2 ; 3$ (needs rev.) \\
\hline
\end{tabular}


Table $1-$ cont.

\begin{tabular}{|c|c|c|}
\hline Umbilicaria deusta (L.) Baumg. & sandstone rocks & $1 ; 2$ \\
\hline Umbilicaria hirsuta (Westr.) Hoffm. & sandstone rocks & $1 ; 2$ \\
\hline Verrucaria caerulea DC. & sandstone rocks & $\begin{array}{l}2 \text { (sub V.glaucina, needs } \\
\text { rev.) }\end{array}$ \\
\hline Verrucaria muralis Ach. & concrete, pebbles & $4(732,784)$ \\
\hline Verrucaria nigrescens Pers. & $\begin{array}{l}\text { tile, concrete, } \\
\text { pebbles }\end{array}$ & 2 (needs rev.); 4 (728) \\
\hline Verrucaria praetermissa (Trevis.) Anzi & stone in stream beds & $4(1043)$ \\
\hline Verrucaria tectorum (A. Massal.) Körb. & sandstone rock & $4(868)$ \\
\hline Verrucaria velana (A. Massal.) Zahlbr. & concrete, stones & 2 \\
\hline Xanthoparmelia conspersa (Ach.) Hale & sandstone rocks & $1 ; 2$ \\
\hline $\begin{array}{l}\text { Xanthoparmelia loxodes (Nyl.) O. Blanco et } \\
\text { al. }\end{array}$ & sandstone rocks & $1 ; 2$ \\
\hline $\begin{array}{l}\text { Xanthoparmelia stenophylla (Ach.) Ahti \& D. } \\
\text { Hawksw. }\end{array}$ & sandstone rocks & $\begin{array}{l}1 ; 2 \text { (sub } X . \text { somolënsis, } \\
\text { needs rev.) }\end{array}$ \\
\hline Xanthoria candelaria (L.) Th.Fr. & $\mathrm{Po}, \mathrm{Fr}$ & $2 ; 4(788)$ \\
\hline Xanthoria elegans (Link.) Th.Fr. & concrete & $2 ; 4(1255)$ \\
\hline Xanthoria fallax (Hepp) Arnold & Po; asbestos tile & $2 ;(793)$ \\
\hline Xanthoria parietina (L.) Th.Fr. & $\begin{array}{l}\text { Po, Ma, Fr, Tc; } \\
\text { concrete }\end{array}$ & $\begin{array}{l}2 ; 4(611,738,769,810,871, \\
889)\end{array}$ \\
\hline Xanthoria polycarpa (Hoffm.) Rieber & Po, Sa, Fr & \\
\hline
\end{tabular}

The list of taxa presented summarizes all reported data (163 species so far) but most of all provides many new regional records. The newly recorded species represent various groups of lichens both in terms of taxonomy and ecology: members of large crustose genera that have been recently revised using modern approaches $-B a$ cidia s.l., Caloplaca, Lecanora, Lepraria, Micarea, Verrucaria; widespread sterile species reported as frequent or common elsewhere, such Fuscidea pusilla, Mycobilimbia epixanthoides, Ropalospora viridis; and endengered species included in the Red List of extinct and threatened lichens in Poland (Cieśliński et al. 2003) - Evernia prunastri, Ramalina farinacea, $R$. fastigiata.

Several of the species deserve special attention. Bacidina sulphurella was reported only from a few sites in Poland until now: Góry Sowie Mts, on Acer pseudoplatanus and Puszcza Knyszyńska Forest, vicinity of Czarna Białostocka, on Carpinus betulus (Brand et al. 2009), and Warszawa city, "Las Bielański” Nature Reserve, on wood (Kubiak et al. 2010). However, it is considered a widespread species in the country (Kubiak et al. 2010). The species represents the B. arnoldiana group, which is distingushed by a finely granular thallus entirely covered by goniocysts, rather large and flat apothecia, with a greyish-brown disc with a slight violet hue, and paler orangebrown and raised margins, and a typically dark brown to red-brown hypothecium reacting $\mathrm{K}+$ dark brown. Revision of the material referred to as $B$. arnoldiana in Western Europe and Macaronesia by Brand et al. (2009) demonstrated that two species can be recognized on the basis of the size of pycnidia and more particularly on the shape of the conidia: B. arnoldiana (Körb.) V. Wirth \& Vězda characterised by filiform, arched or curved (rarely almost straight) conidia, and B. sulphurella characterised by filiform conidia, curved or not, but always with at least one end strongly hooked and slightly enlargered. Moreover, the authors discovered that in addition to morphology the species differ in ecology; the former one is saxicolous and the latter corticolous.

Lecanora albellula [syn. L. piniperda Körb.] was being reported during the last decade with increasing frequency as a result of a broader concept of the species 
presented by Printzen (2001). The species belongs to the L. saligna-related taxa that are characterised by the presence of usnic and/or isousnic acids and corticate amphithecium (traditionally named the L. varia group). Additionally it is distinguished by apothecia rounded to flexuose, usually densely crowded, more rarely single or in small groups, sessile, 0.4-0.6(-0.9) mm diam.; disc light ochre to reddish-brown, matt, finely whitish pruinose, flat to modertely convex; margin weakly prominent when young, persistent or level with disc or often excluded in old apothecia. The most diagnostic character of the species is the apothecial granules that are abundant in the epithecium and densely obscure the whole area of amphithecial cortex. The granules are bright in polarized light and dissolve rapidly in $\mathrm{KOH}$. Lecanora albellula can be mistaken for L. subintricata (Nyl.) Th. Fr. and specimens with dark apothecial discs can also be confused with L. saligna (Printzen 2001). Moreover, the study of the Polish collections of the L. varia group indicated the taxon L. saligna var. sarcopis (Ach.) Hillm. at least pro parte in fact represents L. albellula. The status of the taxa are in urgent need of further investigations.

Lepraria ecorticata has been recently reported for the first time from continental Europe by Kukwa (2006). The author presented records of the species from Poland and the Czech Republic. In Poland Lepraria ecorticata was noted from Równina Bielska Plain, Kaszuby Landlake and Warmia in the north, and from Pogórze Karkonoskie foothills, Góry Sowie Mts, Beskid Wyspowy Mts and Gorce Mts in the south. The species is characterized by a thick, not stratified thallus with most modullary hyphae and soredia well separated from one another. L. ecorticata is very similar in appearance to L. elobata but produces usnic acid in addition to zeorin. It resembles also sorediate species of Lecanora that contain usnic acid as well, e.g., L. expallens, L. compallens Herk \& Aptroot and L. leuckertiana (cf. Kukwa 2006).

Verrucaria praetermissa is an amphibious species occurring exclusively in humid habitats and characterized by a pale green thallus with pinkish white prothallus. The species occupies large siliceous stones emerging above the water surface in streams but occasionally inundated. It is relatively frequently recorded both in lowland and mountainous areas of the country (e.g., Kiszka 1996b; Czarnota 2000; Zalewska 2000; Czyżewska et al. 2001, 2002; Bielczyk 2003; Cieśliński 2003; Krzewicka 2006, 2009).

Verrucaria tectorum is a member of the $V$. nigrescens complex. It is a saxicolous species occupying calcareous rocks and human-made calcium-containing substrata. The species was rarely reported from Poland (Rehman 1879; Kiszka, Kościelniak 1996; Sparrius 2003) but a recent revision of the genus in Poland demonstrated this poorly known species was often mistakenly reported as $V$. nigrescens Pers. (Krzewicka, unpubl.). The quoted treatment yielded numerous new records of the species from the area of the whole country. 


\section{CONCLUSION}

Preliminary inventory of the lichens of the Pogórze Wiśnickie foothills indicates considerable diversity in spite of the high influence of human activity on the landscape and nature of the Carpathians Foothills. Additionally such a large representation of newly reported species belonging to various lichen groups, both in terms of taxonomy and ecology, indicate that further herbarium and field investigations will yield more interesting discoveries. Finally species that urgently require special attention are the lichenicolous fungi. So far only three of the most common species have been reported from the area: Athelia arachnoidea (Stolarczyk 2003), Lichenoconium erodens and L. lecanorae (Stolarczyk 2003; Śliwa, Krzewicka 2004).

Acknowledgements. It is a great pleasure and honour to dedicate this paper to Professor Barbara Gumińska with sincere thanks for her kindness and warm support during my student and academic career at the Institute of Botany of the Jagiellonian University in the years 1986-2003. I am grateful to Beata Krzewicka for friendly and most valuable support during the course of the study. Martin Kukwa (Gdańsk), Paweł Czarnota (Niedźwiedź), Beata Krzewicka and Karina Wilk (Kraków) are thanked for the determination or revising of some critical taxa, Dariusz Kubiak for sharing his ideas and personal comments on taxonomy of Bacidia arnoldiana group, and the following students for accompany me on some field trips: Aneta Sosin, Karina Palka (now Karina Wilk), Barbara Haduch, Michał Węgrzyn, Jerzy Smykla and Piotr Stolarczyk (Kraków). Sincere thanks are due to Krystyna Czyżewska (Łódź) for valuable comments on a paper proposal and to the anonymous reviewer for suggestions on the manuscript. The study was partly supported by the Ministry of Science and Higher Education, grant no. NN 304170539.

\section{REFERENCES}

Bielczyk U. (ed.). 2003. The lichens and allied fungi of the Polish Western Carpathians. The lichens and allied fungi of the Polish Carpathians - an annotated checklist. W. Szafer Institute of Botany, Polish Academy of Sciences, Kraków.

Bielczyk U. 2006. The lichen biota of the Polish Carpathians - General Characteristic. Polish Bot. J. 51 (1): $1-24$.

Brand M., Coppins B., van den Boom P.P.G., Sérusiaux E. 2009. Further data on the lichen genus Bacidia s.l. in the Canary Islands and Western Europe, with descriptions of two new species. Biblioth. Lichenol. 99: 81-92.

Cieśliński S., Fałtynowicz W. (eds). 1993. Atlas of the geographical distribution of lichens in Poland. 1: 1-67. W. Szafer Institute of Botany Polish Academy of Sciences, Kraków.

Cieśliński S., Czyżewska K., Fabiszewski J. 2003. Czerwona lista porostów wymarłych i zagrożonych w Polsce. (In:) K. Czyżewska (ed.), Zagrożenie porostów w Polsce. Monogr. Bot. 91: 13-49.

Cieśliński S. 2003. Atlas rozmieszczenia porostów (Lichenes) w Polsce Północno-Wschodniej. Phytocenosis, 15 (N.S.), Suppl. Cartogr. Geobot. 15: 1-426.

Czarnota P. 2000. Porosty Gorczańskiego Parku Narodowego. Część I. Wykaz i rozmieszczenie gatunków. Parki nar. Rez. Przyr. 19 (1): 3-73.

Czarnota P. 2007. The lichen genus Micarea (Lecanorales, Ascomycota) in Poland. Polish Bot. Stud. 23: $1-199$.

Czwórnóg A., Śliwa L. 1995. Flora porostów rezerwatu "Skamieniałe Miasto" koło Ciężkowic (Pogórze Karpackie). Ochr. Przyr. 52: 185-193.

Czyżewska K. Cieśliński S. Motiejūnaitė J., Kolanko K. 2002. The Budzisk nature reserve as a biocentre of lichen dversity in the Knyszyńska Large Forest (NE Poland). Acta Mycol. 37 (1/2): 77-92.

Czyżewska K. Motiejūnaitė J., Cieśliński S. 2001. Species of lichenised and allied fungi new to Białowieża Large Forest (NE Poland). Acta Mycol. 36 (1): 13-19.

Diederich P., Ertz D., Stapper N., Sérusiaux E., Van Den Broeck D., Van Den Boom P., Ries C. 2010. The lichens and lichenicolous fungi of Belgium, Luxembourg and northern France. Available at http://www.lichenology.info [date of exploration 15 Nov. 2010). 
Drużkowski M. 1998. Współczesna dynamika, funkcjonowanie i przemiany krajobrazu Pogórza Karpackiego, p. 1-285. Instytut Botaniki Uniwersytetu Jagiellońskiego, Kraków.

Kiszka J. 1985. Porosty Pogórza Spiskiego. Studia Ośr. Dokum. Fizjogr. PAN Oddz. Kraków 12: 213 243.

Kiszka J. 1996a. Porosty - wskaźniki gatunkowe skażenia środowiska. (In:) K. Grodzińska, L. Laskowski (eds). Ocena stanu środowiska i procesów zachodzących w lasach zlewni potoku Ratanica (Pogórze Wielickie, Polska Południowa). Państwowa Inspekcja Ochrony Środowiska, Biblioteka Monitoringu Środowiska, Warszawa: 100-104.

Kiszka J. 1996b. Nowe i rzadkie gatunki porostów (Lichenes) w Bieszczadzkim Parku Narodowym. Część I. Roczniki Bieszczadzkie 5: 43-48.

Kiszka J. 2002a. Porosty (Lichenes) oraz warunki bioekologiczne w Arboretum Cisowa (Pogórze Przemyskie). Arboretum Bolestraszyce 9: 47-67.

Kiszka J. 2002b. Notatki o porostach (Lichenes) w Arboretum Bolestraszyce. Część 2. Arboretum Bolestraszyce 9: 41-46.

Kiszka J., Kościelniak R. 1996. Dynamika zmian we florze porostów w obrębie i otoczeniu zbiorników retencyjnych w dolinie Dunajca w Pieninach. (In:) Ogólnopolskie sympozjum na temat: Dynamika zmian środowiska geograficznego pod wpływem antropopresji 'Atmosfera, Hydrosfera-LitosferaCzłowiek', 26-27 września 1996: 55-56, Kraków.

Kiszka J., Piórecki J. 1991. Porosty (Lichenes) Pogórza Przemyskiego. Uniwa, Warszawa.

Kondracki J. 1989. Karpaty. Ed. 2. Wydawnictwa Szkolne i Pedagogiczne, Warszawa.

Kondracki J. 2001. Geografia regionalna Polski. Ed. 2. Wydawnictwo Naukowe PWN, Warszawa.

Kozik R. 1970. Interesujące porosty (Lichenes) Pogórza Rożnowsko-Ciężkowickiego. Rocznik NaukowoDydaktyczny WSP w Krakowie 39, Prace bot. 2: 169-173.

Kozik R. 1976. Lichenoflora dorzecza Białej Dunajcowej. Studia Ośr. Dokum. Fizjogr. PAN Oddz. Kraków 5: 169-196.

Kozik R. 1977. Porosty (Lichenes) Pogórza Rożnowsko-Ciężkowickiego. Fragm. Flor. Geobot. 23 (2): 215-252.

Krzewicka B. 2006. Wodne gatunki porostów z rodzaju Verrucaria w potoku Chochołowskim w Tatrach. (In:) Z. Mirek, B. Godzik (eds). Tatrzański Park Narodowy na tle innych górskich terenów chronionych. Nauki biologiczne, Zakopane: 51-56.

Krzewicka B. 2009. Some new records of Verrucaria from Beskid Niski Mts. Acta Mycol. 44 (2): $265-$ 273.

Krzewicka B., Śliwa L. 2000. Porosty rezerwatu „Prządki” koło Krosna (Pogórze Dynowskie) Ochr. Przyr. 57: 51-58.

Kubiak D., Wrzosek M., Zaniewski P. 2010. Materiały do bioty porostów i grzybów naporostowych rezerwatu „Las Bielański” w Warszawie. Parki nar. Rez. Przyr. 29 (3): 3-15.

Kukwa M. 2006. Notes on taxonomy and distribution of the lichen species Lepraria ecorticata comb. nov. Mycotaxon 97: 63-66.

Leśniański G. 2001. Zagrożone i chronione prawem gatunki porostów na Pogórzu Śląskim. (In:) Botanika w dobie biologii molekularnej. Materiały sesji i sympozjów 52. Zjazdu Polskiego Towarzystwa Botanicznego, p. 176. Wydawnictwo Poznańskie, Poznań.

Orange A., James P. W., White F. J. 2001. Microchemical methods for the identification of lichens. British Lichen Society, London.

Printzen C. 2001. Corticolous and lignicolous species of Lecanora (Lecanoraceae, Lecanorales) with usnic and isousnic acid in the Sonoran Desert region. Bryologist 104: 382-409.

Rehman A. 1879. Systematyczny przegląd porostów znalezionych dotąd w Galicyi zachodniej opracowany na podstawie własnych i cudzych spostrzeżeń Spraw. Kom. Fizyogr. Akad. Umiejętn. 13 (2): 1-66.

Rydzak J. 1955. Wpływ małych miast na florę porostów. Cz. II. Beskidy Zachodnie. Wisła, Ustroń, Muszyna, Iwonicz, Rymanów, Lesko. Ann. Univ. Mariae Curie-Skłodowska, Sect. C, Biol. 10 (2): 33-66.

Santesson R., Moberg R., Nordin A., Tønsberg T. Vitikainen O. 2004. Lichen-forming and lichenicolous fungi of Fennoscandia, Uppsala.

Sparrius L. 2003. Contribution to the lichen flora of the Białowieża Forest and Biebrza valley (Eastern Poland). Herzogia 15: 155-160.

Stachurska A. 1998a. Zbiorowiska leśne północno-wschodniej części Pogórza Wielickiego (Zachodnie Karpaty). Zesz. Nauk. Uniw. Jagiell., Prace Bot. 30: 1-78. 
Stachurska A. 1998b. Forest communities and their transformations in the benchland area of the Carpathian foreland. Prace Geogr. Inst. Geogr. UJ 103: 179-188.

Stolarczyk P. 2003. Porosty Wiśnicko-Lipnickiego Parku Krajobrazowego na Pogórzu Wiśnickim. Fragm. Flor. Geobot. Polonica 10: 241-252.

Śliwa L., Krzewicka B. 2004. Lichens of the Bukowiec Nature Reserve (Pogórze Wiśnickie Foothills). Fragm. Flor. Geobot. Polonica 11: 171-175.

Śliwa L., Krzewicka B., Sosin A., Stolarczyk P. 2001. Lichens of the protected sandstone tors in Pogórze Wiśnickie (Wiśnickie Foothills, Carpathians). Chrońmy Przyr. Ojczystą 57 (3): 32-42.

Zalewska A. 2000. Ekologia porostów Puszczy Boreckiej i jej obrzeży. PhD thesis, Olsztyn.

\section{Materiały do bioty porostów Pogórza Wiśnickiego (Karpaty)}

\section{Streszczenie}

W latach 1998-2000 przeprowadzono badania lichenologiczne na obszarze Pogórza Wiśnickiego (Karpaty Zachodnie) ze szczególnym uwzględnieniem obszarów chronionych. Praca ta jest pierwszą z planowanych prac podsumowujących wyniki tych badań. Warto podkreślić, że Pogórze Karpackie to bardzo interesujący obiekt do badań, który pozostając pod znacznym wpływem działalności człowieka zachował wiele cennych składników przyrodniczych.

Obecna lista porostów Pogórza Wiśnickiego liczy 163 gatunki, wiele z nich podano z tego terenu po raz pierwszy. Gatunki nowe reprezentują różne grupy porostów, interesujące z taksonomicznego i ekologicznego punktu widzenia. Są to przedstawiciele licznych w gatunki rodzajów skorupiastych, które doczekały się w ostatnim czasie nowoczesnych rewizji - Bacidia s.1., Caloplaca, Lecanora, Lepraria, Micarea, Verrucaria; rozpowszechnione gatunki porostów występujących w stanie płonnym - Fuscidea pusilla, Mycobilimbia epixanthoides i Ropalospora viridis oraz gatunki, które znalazły się na liście porostów zagrożonych w Polsce (Cieśliński et al. 2003) - Evernia prunastri, Ramalina farinacea, $R$. fastigiata. Na szczególną uwagę zasługują Bacidina sulphurella, Lecanora albellula, Lepraria ecorticata, Verrucaria praetermissa i V. tectorum. 